\title{
Un año de pacientes nuevos en un Servicio de Salud Mental
}

\begin{abstract}
RESUMEN: Muestra con 1.406 pacientes nuevos en el Servicio de Salud Mental de Aranjuez (2004), para realizar un perfil sociodemográfico de los pacientes nuevos vistos durante ese año, y conocer la distribución de las categorías diagnósticas totales y en función de las variables sexo y edad.

PALABRAS CLAVE: Servicios de Salud Mental, atención ambulatoria, epidemiología psiquiátrica.
\end{abstract}

SUMMARY: A total of 1.406 new patients were reviewed in the Mental Health Service of Aranjuez (2004). We want to make a socio-demographic profile of the new patients and, to know the distribution of the diagnostic categories and related to sex and age variables.

KEY WORDS: Mental Health Services, ambulatory care, psychiatric epidemiology.

El Servicio de Salud Mental que ha venido atendiendo a la población del distrito sanitario de Aranjuez (Madrid) desde 1986 se ha basado en la colaboración entre la Comunidad de Madrid y la Congregación de Hermanas Hospitalarias del Sagrado Corazón de Jesús (Complejo Asistencial Benito Menni). Esta colaboración ha adoptado varias modalidades administrativas a lo largo del tiempo, siendo la última la modalidad de concierto público. Esta situación se ha mantenido hasta la finalización del último concierto en diciembre de 2005.

El Servicio de Salud Mental de Aranjuez atendía a la población del distrito sanitario de Aranjuez, incluida en el Área sanitaria número 11 de la Comunidad Autónoma de Madrid. Como se observa en la figura 1, esta área está formada por dos sectores geográficos muy diferenciados. Existe un territorio de carácter urbano, situado en el sur del municipio de Madrid. El segundo sector comprende el distrito sanitario de Aranjuez propiamente dicho y engloba las comarcas de las vegas de los ríos Jarama, Tajo y Tajuña y está compuesto por un conjunto de municipios de carácter semiurbano y rural. La población de este distrito ascendía a 115.471 habitantes según el último censo de 2001, cifra que en el 2004 ha sido claramente superada (a 30 de abril de 2005 el número de tarjetas individuales sanitarias, según datos de la Gerencia de Atención Primaria del Área 11 se sitúa en torno a 150.000). En la tabla 1 se recogen las principales características sociodemográficas de este distrito y su comparativa con respecto al total de población de la Comunidad de Madrid (1).

Se trata de un distrito semirural, salvo el municipio de Valdemoro que se puede considerar incluido dentro del área de influencia de Madrid, y el municipio de Aranjuez, caracterizado por una actividad volcada en el sector servicios. Es una 
Figura 1

Área 11 y Distrito Sanitario de Aranjuez (Memoria de Atención Primaria, 2003)

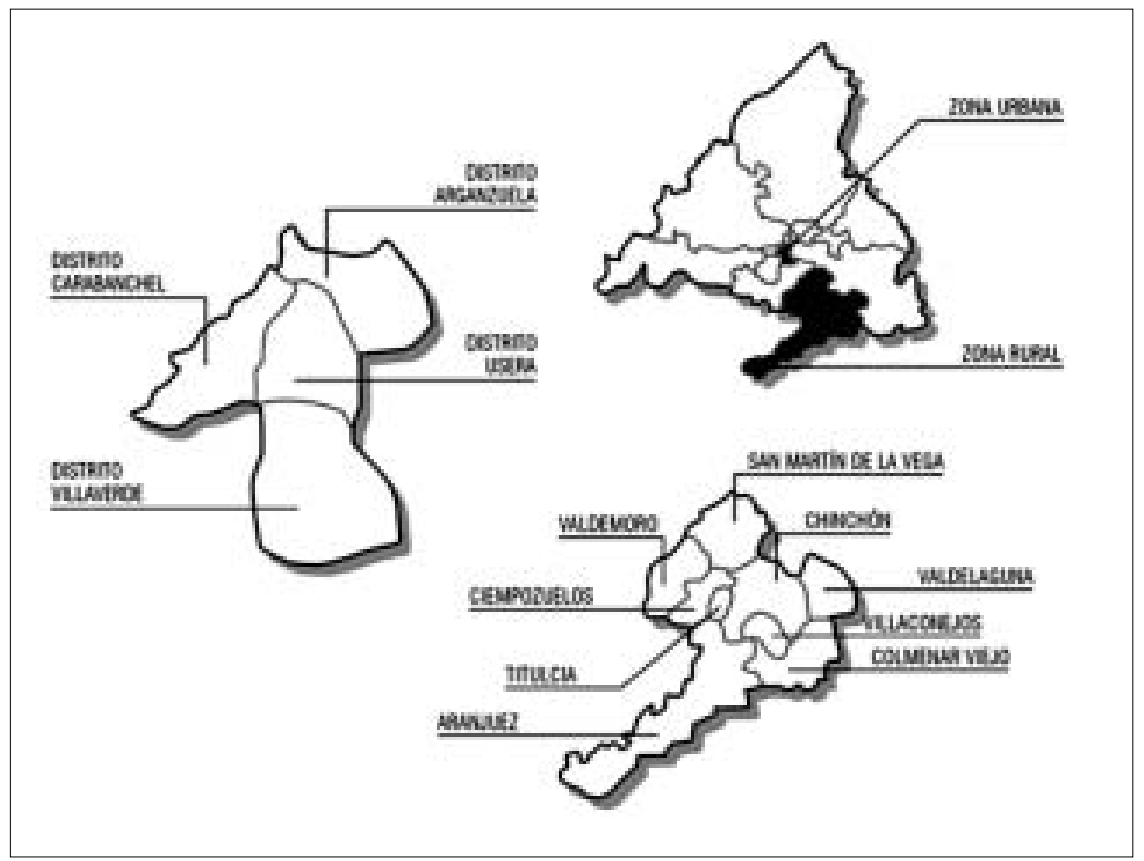

población más joven que el resto de la Comunidad de Madrid. El nivel socioeconómico está por debajo del promedio de la Comunidad de Madrid. El nivel educativo es también inferior, con mayor proporción de analfabetismo y carencia de estudios básicos. La proporción de población con estudios superiores es escasa en comparación con el resto del territorio de la Comunidad.

Es un distrito de una gran extensión y dispersión. Algunos municipios distan hasta 25-30 Km del punto asistencial más cercano del servicio, con una mala oferta de transporte público. Por este motivo, de cara a mejorar la accesibilidad de la población, la actividad asistencial del Servicio se llevaba a cabo en tres Dispositivos asistenciales: Aranjuez (dispositivo principal y cabecera del distrito), Valdemoro y Ciempozuelos. Cada una de estas sedes atendía a su población de referencia de acuerdo al siguiente esquema:

Dispositivo principal (Aranjuez): Atendía dos zonas básicas de Salud (Aranjuez y Colmenar de Oreja), con tres Equipos de Atención Primaria (Aranjuez I y II, Colmenar de Oreja) cubriendo los siguientes municipios: Aranjuez, Colmenar de Oreja, Chinchón, Valdelaguna, Villaconejos. 
Tabla 1

Datos sociodemográficos del Distrito de Aranjuez y de la Comunidad de Madrid

\begin{tabular}{|c|c|c|}
\hline & Distrito Aranjuez & Comunidad de Madrid \\
\hline N. ${ }^{\text { habitantes (2004) }}$ & 130.012 & 5.964 .143 \\
Varones (\%) & 50,08 & 48,42 \\
Mujeres (\%) & 49,9 & 51,57 \\
\hline Edad (\%) (Censo 2001) & & \\
< 15 años & 17,51 & 14,29 \\
15-64 años & 69,91 & 71,15 \\
$>$ 64 años & 12,57 & 14,55 \\
\hline Desempleo (\%) & 3,31 & 3,46 \\
\hline Ratio desempleo & 1,79 & 1,40 \\
Mujer / Varón & & \\
\hline Actividad profesional (\%) & 5,54 & 0,80 \\
Agrario & 19,93 & 13,51 \\
Industria & 18,37 & 9,61 \\
Construcción & 56,14 & 76,08 \\
\hline Servicios & $9.047,76$ & $13.094,11$ \\
\hline Renta Per cápita (€) & & \\
\hline Nivel educativo (\%) & 7,59 & 20,74 \\
Tercer grado & 48,26 & 50,09 \\
Segundo grado & 24,29 & 17,44 \\
Primer grado & 16,04 & 9,97 \\
Sin estudios & 3,79 & 1,77 \\
Analfabeta & & \\
\hline
\end{tabular}

Dispositivo de Valdemoro: Atendía a la Zona Básica de Salud de Valdemoro, prestando asistencia a la población del municipio de Valdemoro, que incluye dos equipos de Atención Primaria.

Dispositivo de Ciempozuelos: Atendía dos zonas básicas de Salud (Ciempozuelos y San Martín de la Vega) cubriendo los municipios de Ciempozuelos, Titulcia y San Martín de la Vega. Incluye dos equipos de Atención Primaria.

La Comunidad de Madrid está organizada, sanitariamente, en once áreas de salud, cada una de estas áreas se subdivide en varios distritos sanitarios. Cada distrito consta de un Servicio de Salud Mental, que se encarga de la atención ambulatoria de todos los problemas psiquiátricos y de salud mental de toda la población de referencia. El funcionamiento de los Servicios de Salud Mental es el propio de la Atención Especializada, siendo su principal ruta asistencial la procedente de 
Atención Primaria, si bien es posible la derivación de casos desde los diferentes especialistas, atención hospitalaria, así como otras instancias no sanitarias (Servicios Sociales, Equipos de Orientación Psicopedagógicos, Administración de Justicia, etc.).

El modelo que fundamenta estos Servicios de Salud Mental es el de la Psiquiatría Comunitaria (2). Los Servicios están constituidos por equipos multidisciplinares, integrados por psiquiatras, psicólogos/as clínicos, trabajadores/as sociales, enfermero/a y administrativo/a. En estos Servicios se actúa mediante programas de obligada implantación por la Comunidad de Madrid (3). Estos programas cubrirían las diferentes necesidades asistenciales detectadas en un determinado territorio: patología infantil y juvenil, atención ambulatoria, psicogeriatría, rehabilitación, alcoholismo, etc.

Los diferentes Servicios de Salud Mental actúan en coordinación con una red de dispositivos asistenciales de Salud Mental (dispositivos de rehabilitación, hospitalización y socio comunitarios) localizados en cada una de las áreas sanitarias. Esta distribución no está al mismo nivel en todas las áreas de salud, encontrándose en vías de desarrollo. En el Servicio de Salud Mental de Aranjuez se contaba, en el año 2004, con los siguientes dispositivos:

Dispositivos de Rehabilitación: Centros de Rehabilitación Psicosocial «Los Cármenes» y «Nuestra Señora del Sagrario»; Centro de Rehabilitación Laboral; Mini-Residencia «Carabanchel». Estos tres últimos dispositivos se sitúan en el Municipio de Madrid. Unidades de Media y Larga Estancia (Complejo Asistencial Benito Menni y Hospital San Juan de Dios) situados en el municipio de Ciempozuelos; Centros de Día de Aranjuez, Valdemoro y Mancomunidad de la Vega (Ciempozuelos). A finales del año 2004 comenzó a funcionar un Centro de Rehabilitación Psicosocial y un Centro de Día en el Municipio de Aranjuez.

Dispositivos de soporte social: pisos supervisados, situados en el municipio de Madrid. Otros recursos: Parroquias, ONG, Asociaciones, etc.

Recursos socio comunitarios: son compartidos con el resto de la población (Atención Primaria, Especializada, recursos educativos, residenciales, de empleo, etc.).

Dispositivos de hospitalización: En el caso de la población adulta, las Unidades de Hospitalización Breve de referencia son las ubicadas en el H. U. «12 de Octubre» (Madrid) y el «Complejo Asistencial Benito Menni» (Ciempozuelos). Para la población infanto-juvenil los hospitales de referencia son Hospital «Niño Jesús» (Madrid) y la Unidad de Adolescentes del H. G. U. Gregorio Marañón (Madrid). Unidad de Deshabituación alcohólica (situada al Norte del Municipio de Madrid). 
Centro Comarcal de Drogodependencias (Aranjuez) y Agencia Antidroga: La sectorización de la Comunidad de Madrid establece que la patología por drogodependencia es atendida en una ruta asistencial diferente a Salud Mental, excepto en los casos de alcoholismo. Esta distinción es difícil de mantener en la práctica diaria de ambas redes.

Otros dispositivos, como los hospitales de día, se han ido desarrollando a partir del 2004. Con anterioridad estaban disponibles los situados en otras áreas de Madrid, geográficamente muy inaccesibles para nuestra población.

Respecto a los recursos humanos del propio Servicio de Salud Mental, en el año 2004 el equipo estaba constituido por cinco psiquiatras, dos psicólogos a tiempo completo y uno a tiempo parcial, dos enfermeras, una trabajadora social, dos administrativas a tiempo completo y una a tiempo parcial. Para la atención a la población infanto-juvenil del distrito (hasta los 18 años), de los recursos mencionados, se destinaban un psiquiatra y una psicóloga clínica a tiempo completo como subequipo específico. El resto de actuaciones profesionales (enfermería, trabajo social, administración, etc.) eran compartidas con el resto de programas y tareas asistenciales.

La actividad asistencial del Servicio e indicadores, así como los índices de asistencia / caso para los principales diagnósticos, durante el año 2004, figuran en las tablas 2 y 3 respectivamente.

Durante el año 2004 se ha producido nuevamente un incremento en el número de pacientes nuevos, pacientes en contacto e indicadores de actividad asistencial, como se puede apreciar en la figura 2.

Tabla 2

Indicadores de actividad asistencial del S. S. M. de Aranjuez, (2004)

\begin{tabular}{|l|r|r|r|r|r|r|r|r|r|}
\hline & \multicolumn{3}{|c|}{ Hombres } & \multicolumn{3}{c|}{ Mujeres } & \multicolumn{3}{c|}{ Total } \\
\hline & $<16$ & $>64$ & \multicolumn{1}{|c|}{ Total } & $<16$ & $>64$ & Total & $<16$ & $>64$ & Total \\
\hline Población (Censo 2001) & 11.087 & 6.098 & 57.350 & 10.500 & 8.418 & 58.121 & 21.587 & 14.516 & 115.471 \\
\hline Pacientes en contacto & 345 & 129 & 1.497 & 217 & 373 & 2.333 & 562 & 502 & 3.830 \\
\hline Casos nuevos & 185 & 33 & 594 & 141 & 80 & 809 & 326 & 113 & 1.403 \\
\hline Asistencias totales & 1.584 & 469 & 8.255 & 778 & 1.413 & 11.776 & 2.362 & 1.882 & 20.031 \\
\hline Incidencia & 16,69 & 5,41 & 10,36 & 13,43 & 9,50 & 13,92 & 15,10 & 7,78 & 12,15 \\
\hline Frecuentación & 117,71 & 64,12 & 120,00 & 61,05 & 136,97 & 167,07 & 90,15 & 106,37 & 143,69 \\
\hline Prevalencia & 31,12 & 21,15 & 26,10 & 20,67 & 44,31 & 40,14 & 26,03 & 34,58 & 33,17 \\
\hline
\end{tabular}


Tabla 3

Casos atendidos por diagnósticos (2004)

\begin{tabular}{|l|r|r|r|}
\hline \multicolumn{1}{|c|}{ Diagnóstico } & Casos & Asistencias & Asist./caso \\
\hline Esquizofrenia y psicosis paranoides & 164 & 1.883 & 11,48 \\
\hline Psicosis afectivas & 209 & 1.133 & 5,42 \\
\hline Otros trastornos depresivos & 850 & 2.927 & 3,44 \\
\hline Alcoholismo y trastornos por tóxicos & 107 & 323 & 3,02 \\
\hline Otros trastornos neuróticos & 545 & 1.939 & 3,56 \\
\hline Demencias y psicosis orgánicas & 31 & 98 & 3,16 \\
\hline Otros trastornos mentales orgánicos & 23 & 100 & 4,35 \\
\hline Trastornos de la personalidad & 106 & 616 & 5,81 \\
\hline Otros & 1.769 & 7.534 & 4,25 \\
\hline Sin especificar & 9 & 12 & 1,33 \\
\hline
\end{tabular}

Figura 2

Evolución del S. S. M. de Aranjuez desde su creación
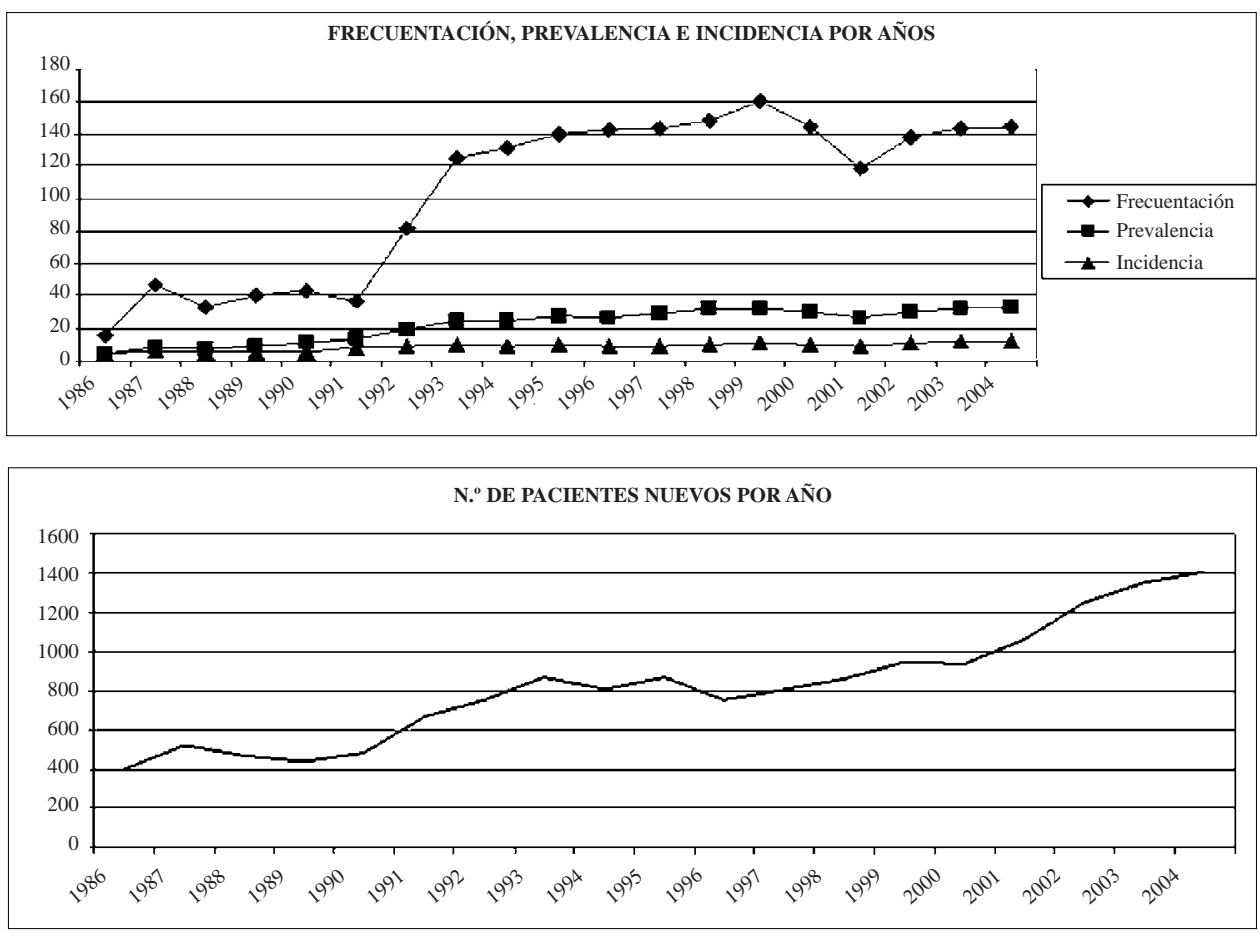
En el año 2004 tuvo lugar el atentado terrorista conocido como 11-M. El nivel de afectación sobre este distrito fue muy bajo. Los datos reflejados en este trabajo no incluyen los casos diagnósticos catalogados como afectados por el $11-\mathrm{M}$.

Estos indicadores son generados por el programa de Registro Acumulado de Casos. La incidencia recoge el número de casos nuevos por mil habitantes; respecto a la prevalencia, consiste en el número de casos nuevos y antiguos por mil habitantes en contacto con el servicio. La frecuentación señala el número de consultas realizadas por mil habitantes. La actualización de las cifras poblacionales modifica estos indicadores, circunstancia que se produce coincidiendo con los censos oficiales (último en el año 2001). A pesar de este ajuste corrector, como se puede observar en la figura, se aprecia un constante incremento desde la creación del Servicio, en consonancia con las características demográficas del distrito (zona de la Comunidad de Madrid en expansión urbanística, receptora de nueva población).

Con posterioridad a la realización de este trabajo, el Servicio de Salud Mental del distrito sanitario de Aranjuez ha experimentado notables cambios. En el año 2006 la población del municipio de Valdemoro comenzó a ser atendida por un Servicio de Salud Mental independiente y de gestión pública. El resto del distrito sanitario sigue siendo atendido bajo gestión privada (modalidad de concierto) pero por dos empresas diferentes: el dispositivo de Ciempozuelos continúa a cargo del Complejo Asistencial Benito Menni (Hermanas Hospitalarias del Sagrado Corazón de Jesús) y el dispositivo de Aranjuez es gestionado por el Hospital San Juan de Dios de Ciempozuelos (Hermanos de San Juan de Dios).

\section{Objetivos del estudio}

Este trabajo tiene como objetivo principal la descripción de los pacientes nuevos vistos en el Servicio de Salud Mental de Aranjuez durante el año 2004. Este análisis descriptivo ha incluido variables sociodemográficas, como sexo, edad, estado civil, situación laboral, ocupación, convivencia, nivel educativo y procedencia. Este nivel de análisis permitirá la realización de un perfil sociodemográfico del paciente de este distrito en el año 2004.

Por otra parte, se pretende conocer los diagnósticos principales realizados, así como la distribución de los mismos en función del sexo y la edad. 


\section{Material y métodos}

Los pacientes, objeto de este estudio, son derivados fundamentalmente desde los equipos de Atención Primaria del distrito y, en menor medida, de Atención Especializada. Es importante señalar que los problemas relacionados con el consumo de tóxicos, con la excepción del alcohol, tienen una ruta asistencial diferenciada. Son remitidos mayoritariamente desde Atención Primaria al Centro Comarcal de Drogodependencias, situado en Aranjuez. Hay una parte importante de psicopatología relacionada con el alcohol (patología dual) que es probable que no sea vista en este SSM, dado el patrón habitual de policonsumo.

El SSM de Aranjuez, como todos los SSM de distrito de la Comunidad de Madrid, cuenta con un programa informático para el registro de datos sociodemográficos, diagnósticos y asistenciales que permite el seguimiento y control de la actividad del Servicio. Este programa se conoce como Registro Acumulado de Casos Psiquiátricos (RAC); es proporcionado y controlado por la Oficina Regional de Coordinación de Salud Mental.

Los datos que se introducen en este RAC proceden de varias fuentes:

Hoja de Identificación del Paciente: Se cumplimenta en el momento de la recepción del paciente en el Servicio e incluye los siguientes datos: identificación del paciente, domicilio, estado civil, tipo de convivencia, nivel educativo, procedencia, profesión, situación laboral y, en el caso de niños y adolescentes, escolarización y datos del padre/madre. Este protocolo es cumplimentado por personal administrativo y se lleva a cabo en el mostrador de recepción, con escasa intimidad en la transmisión de los datos, que es realizada de forma oral.

Ficha de Asistencia: Se cumplimenta tras cada acto asistencial con el paciente, recogiendo los siguientes datos: tipo de prestación recibida, modalidad de atención, grupo de edad y diagnósticos según la codificación de la novena edición de la Clasificación Internacional de Enfermedades de la Organización Mundial de la Salud (4). Este protocolo es realizado por los profesionales clínicos (psiquiatras y psicólogos clínicos).

Este programa informático proporciona una serie limitada de listados para el análisis de la actividad asistencial. Estos listados han constituido la base para la elaboración de este trabajo, no siendo posible la obtención de todas las combinaciones de datos posibles.

De acuerdo con este programa, se considera paciente nuevo a toda persona que nunca antes había tenido contacto con el Servicio, por lo que quedan excluidos de la consideración de nuevos aquellas personas vistas durante el 2004 que 
tuvieran un contacto previo. Estos casos no son considerados como nuevos por el programa, aunque hayan pasado años.

El análisis de este trabajo se centra en los pacientes nuevos vistos en el año 2004 según la consideración de este programa informático, y atendiendo al primer diagnóstico codificado en la primera consulta. La falta de un diagnóstico definido en la primera consulta se consignaba como diagnóstico sin especificar.

El estadístico descriptivo utilizado ha sido la proporción, expresada en tanto por ciento, en relación a la variable correspondiente. Se ha elegido la proporción frente a los datos directos, por permitir las comparaciones entre niveles de la variable (edades, sexo, ocupación, etc.).

Las categorías diagnósticas consideradas han sido los epígrafes generales de la Novena Edición de la Clasificación Internacional de Enfermedades de la Organización Mundial de la Salud (4). Esta novena edición (1977) es la que permanece vigente en la Red de Salud Mental de la Comunidad de Madrid. El conjunto de categorías diagnósticas empleadas puede consultarse en la tabla 4. En algunas categorías se han desglosado los datos porque entendemos que ofrecen un mejor análisis, por ejemplo: se ha convertido al diagnóstico de Abuso de alcohol en una categoría principal (305.0), se ha desglosado la categoría de Síndromes especiales no clasificados en otra parte (307) y se han unificado las diferentes categorías de retraso mental.

\section{Resultados}

Los datos analizados permiten la descripción sociodemográfica de los pacientes nuevos. En la tabla 5 se presentan los datos referidos a las variables que se incluyen en la Hoja de Identificación de Datos. La edad media de la muestra es de 32,65 años, siendo la mediana de 31 años, siendo congruente con las características de la población del distrito (tabla 1).

En la tabla 6 se muestra un análisis sociodemográfico en el que se tiene en cuenta la variable sexo pero no sobre todas las variables de la Hoja de Identificación, ya que el programa informático de este SSM no proporciona dicha información referida a situación laboral y ocupación.

Con respecto al análisis de los diagnósticos, en la tabla 7 se recoge la distribución, expresada en porcentaje, por sexos y totales. En la tabla 8 se muestra la distribución de los diferentes diagnósticos en función de seis grupos etarios. Esta estructura de seis grupos viene impuesta por el programa informático de este SSM. 
Tabla 4

Categorías diagnósticas principales según C.I.E. -9- O.M.S

\begin{tabular}{|c|c|}
\hline 290 & Psicosis orgánica senil y presenil \\
\hline 291 & Psicosis alcohólicas \\
\hline 292 & Psicosis debidas a drogas \\
\hline 293 & Psicosis orgánicas transitorias \\
\hline 294 & Otras psicosis orgánicas (crónicas) \\
\hline 295 & Psicosis esquizofrénica \\
\hline 296 & Psicosis afectivas \\
\hline 297 & Estados paranoides \\
\hline 298 & Otras psicosis no orgánicas \\
\hline 299 & Psicosis peculiares de la niñez \\
\hline 300 & Trastornos neuróticos \\
\hline 301 & Trastornos de la personalidad \\
\hline 302 & Desviaciones y trastornos sexuales \\
\hline 303 & Síndrome de dependencia del alcohol \\
\hline 304 & Dependencia de las drogas \\
\hline 305.0 & Alcohol (abuso) \\
\hline 305 & Abuso de drogas sin dependencia \\
\hline 306 & Alteraciones corporales originadas por factores mentales \\
\hline 307 & $\begin{array}{l}\text { Síntomas o síndromes especiales no clasificados en otra parte } \\
\text { Movimiento } \\
\text { Alimentación } \\
\text { Sueño } \\
\text { Esfínteres } \\
\text { Dolor }\end{array}$ \\
\hline 308 & Reacción aguda ante gran tensión \\
\hline 309 & Reacción de adaptación \\
\hline 310 & Trastornos mentales no psicóticos por lesión orgánica encefálica \\
\hline 311 & Trastornos depresivos no clasificados en otra parte \\
\hline 312 & Perturbación conducta no clasificada en otra parte \\
\hline 313 & Perturbación de las emociones propias de la niñez y adolescencia \\
\hline 314 & Síndrome hipercinético de la niñez \\
\hline 315 & Retardo selectivo del desarrollo \\
\hline 316 & $\begin{array}{l}\text { Factores psíquicos asociados con enfermedades clasificadas en otra parte } \\
\text { Retraso mental } \\
\text { Sin especificar } \\
\text { No patología } \\
\text { Códigos V }\end{array}$ \\
\hline
\end{tabular}


Tabla 5

Datos sociodemográficos totales de la muestra clínica

\begin{tabular}{|c|c|c|c|c|c|}
\hline PROCEDENCIA & $\%$ & $\begin{array}{l}\text { CONVIVENCIA } \\
\text { MENORES } \\
(<17 \text { años })\end{array}$ & $\%$ & OCUPACIÓN & $\%$ \\
\hline Valdemoro & 35,6 & Con ambos padres & 77,4 & \multirow{2}{*}{$\begin{array}{l}\text { Ocupaciones no bien } \\
\text { especificadas }\end{array}$} & \multirow{2}{*}{42,5} \\
\hline Aranjuez & 33,3 & Sólo con madre & 20,9 & & \\
\hline Ciempozuelos & 11,4 & Sólo con padre & 0,9 & \multirow{2}{*}{$\begin{array}{l}\text { Hostelería, servicios } \\
\text { y seguridad }\end{array}$} & \multirow{2}{*}{36,8} \\
\hline San Martín de la Vega & 10,6 & Con otros familiares & 0,6 & & \\
\hline Chinchón & 3,2 & \multirow{2}{*}{\multicolumn{2}{|c|}{ En institución }} & Comerciantes y & \multirow[b]{2}{*}{8,9} \\
\hline Villaconejos & 2,3 & & & vendedores & \\
\hline Colmenar de Oreja & 2,2 & \multirow{2}{*}{\multicolumn{2}{|c|}{$\begin{array}{c}\text { CONVIVENCIA } \\
\text { ADULTOS (> } 16 \text { años) }\end{array}$}} & Agricultura y & \\
\hline Otras & 0,4 & & & ganadería & 4,3 \\
\hline Valdelaguna & 0,01 & Con cónyuge & 52,6 & Profesionales y & 2,8 \\
\hline \multicolumn{2}{|l|}{ EDADES } & $\begin{array}{l}\text { Con padres } \\
\text { Solo }\end{array}$ & $\begin{array}{r}16,6 \\
8,6\end{array}$ & $\begin{array}{l}\text { tecnicos } \\
\text { Personal servicios }\end{array}$ & 2.5 \\
\hline $31-45$ & 30,6 & Con pareja & 6,8 & administrativos & \\
\hline $0-15$ & 24,7 & Con hijos & 5,1 & Personal industria, & \\
\hline $16-30$ & 21,8 & En institución & 3,8 & construcción y & 1 \\
\hline $46-60$ & 14,1 & Sólo con madre & 3,7 & $\operatorname{trans}$ & \\
\hline $61-75$ & 6 & Con otros familiares & 2 & Directivos y gerentes & 1 \\
\hline $75-$ & 3,3 & $\begin{array}{l}\text { Sólo con padre } \\
\text { Otros }\end{array}$ & $\begin{array}{l}0,5 \\
0,4\end{array}$ & \multicolumn{2}{|c|}{ NIVEL EDUCATIVO } \\
\hline SEXO & & Sin especificar & 0,1 & Certificado de & 52,4 \\
\hline $\begin{array}{l}\text { Mujeres } \\
\text { Varones }\end{array}$ & $\begin{array}{l}57,6 \\
42,4\end{array}$ & \multicolumn{2}{|c|}{ SITUACIÓN LABORAL } & $\begin{array}{l}\text { estudios } \\
\text { Sin especificar }\end{array}$ & 21,5 \\
\hline \multirow{2}{*}{\multicolumn{2}{|c|}{ ESTADO CIVIL }} & $\begin{array}{l}\text { Retirado, jubilado, } \\
\text { pensionista }\end{array}$ & 45,1 & $\begin{array}{l}\text { Educación General } \\
\text { Básica }\end{array}$ & 11,7 \\
\hline & & Labores hogar & 21,2 & Sin estudios & 5,4 \\
\hline $\begin{array}{l}\text { Soltero/a } \\
\text { Casado/a }\end{array}$ & $\begin{array}{l}49 \\
417\end{array}$ & Trabajando & 17,5 & B & 3,8 \\
\hline Casado/a & $\begin{array}{r}41,7 \\
43\end{array}$ & Escolar & 13,2 & Doctorado & 2,8 \\
\hline $\begin{array}{l}\text { Viudo/a } \\
\text { Separado/a }\end{array}$ & $\begin{array}{l}4,3 \\
3,9\end{array}$ & Parado (no primer & 09 & $\begin{array}{l}\text { Analfabeto } \\
\text { Universitario/a }\end{array}$ & 1,4 \\
\hline Divorciado/a & $\begin{array}{l}5,9 \\
0,8\end{array}$ & empleo) sin subsidio & 0,9 & $\begin{array}{r}\text { (Primer ciclo) } \\
\text { (Priofia }\end{array}$ & 0,6 \\
\hline Sin especificar & 0,1 & $\begin{array}{l}\text { transitoria } \\
\text { Busca } \\
\text { primer empleo } \\
\text { Sin especificar }\end{array}$ & $\begin{array}{l}0,5 \\
0,3\end{array}$ & $\begin{array}{l}\text { Universitario/a } \\
\text { (Segundo ciclo) }\end{array}$ & 0,3 \\
\hline
\end{tabular}


Tabla 6

Datos sociodemográficos de la muestra clínica en función del sexo

\begin{tabular}{|c|c|c|c|c|c|}
\hline \multirow[t]{2}{*}{ PROCEDENCIA } & \multicolumn{2}{|c|}{$\%$} & \multirow{2}{*}{$\begin{array}{c}\text { CONVIVENCIA } \\
\text { (Mayores de } 17 \text { años) }\end{array}$} & \multicolumn{2}{|c|}{$\%$} \\
\hline & $\mathbf{V}$ & $\mathbf{M}$ & & $\mathbf{V}$ & M \\
\hline Aranjuez & 32,4 & 34 & Con cónyuge & 50,2 & 54 \\
\hline Ciempozuelos & 12,9 & 10,3 & Con padres & 17,6 & 16 \\
\hline San Martín de la Vega & 10,2 & 10,8 & Solo & 11,4 & 6,8 \\
\hline Valdemoro & 33,7 & 37 & Con pareja & 5,2 & 7,8 \\
\hline Colmenar de Oreja & 2,6 & 1,9 & Con hijos & 2,5 & 6,7 \\
\hline Titulcia & 1 & 0,3 & En institución & 4 & 3,7 \\
\hline Villaconejos & 2,6 & 2,1 & Sólo con madre & 5,7 & 2,4 \\
\hline Chinchón & 3,7 & 2,8 & Con otros familiares & 2,7 & 1,5 \\
\hline Valdelaguna & 0 & 0,1 & Sólo con padre & 0,5 & 0,5 \\
\hline Otras procedencias & 0,6 & 0,2 & Otras modalidades & 0,2 & 0,5 \\
\hline \multicolumn{4}{|l|}{ EDAD } & & 0,2 \\
\hline $0-15$ & 32 & 19 & NIVEL EDUCATIVO & & \\
\hline $16-30$ & 17,1 & 24,3 & Certificado de estudios & 48,9 & 54,9 \\
\hline $31-45$ & 29,2 & 31,8 & Sin especificar & 27,7 & 16,9 \\
\hline $46-60$ & 14,7 & 13,7 & Educación General Básica & 11,7 & 11,6 \\
\hline $61-75$ & 4,5 & 7 & Sin estudios & 3,7 & 6,7 \\
\hline 76- & 2,3 & 4 & Bachillerato & 3,2 & 4,3 \\
\hline & & & Doctorado & 2,3 & 3,2 \\
\hline ESTADO CIVIL & & & Analfabeto & 1,5 & 1,2 \\
\hline Soltero/a & 58,2 & 42,2 & Universitario/a & 0,3 & 0,9 \\
\hline Casado/a & 35,7 & 46,1 & $\begin{array}{l}\text { (Primer ciclo) } \\
\text { Universitariola }\end{array}$ & & \\
\hline Viudo/a & 1,8 & 6,1 & $\begin{array}{l}\text { Universitario/a } \\
\text { (Segundo ciclo) }\end{array}$ & 0,3 & 0,2 \\
\hline Separado/a & 3,8 & 4,1 & $\begin{array}{l}\text { (Segundo ciclo) } \\
\text { Títulos no clasificables }\end{array}$ & & \\
\hline Divorciado/a & 0,5 & 1,1 & por grados & 0,2 & - \\
\hline Sin especificar & 0 & 0,2 & & & \\
\hline
\end{tabular}

\section{Comentario y discusión}

Antes de lanzarnos a adelantar conclusiones a partir de los resultados obtenidos, creemos conveniente advertir sobre los inevitables sesgos que son inherentes al tipo de información que se maneja, así como las fuentes de las que se obtiene. En otros trabajos similares $(5 ; 6)$ ya se advierte de estos problemas metodológicos, de amplio espectro, pues la información se recoge por diferentes profesionales, no clínicos algunos de ellos, en condiciones de escasa o nula confidencialidad. En el caso de los clínicos, existen diferencias en formación, 
hábitos diagnósticos, orientación terapéutica, que condicionan el proceso de evaluación diagnóstica. Otro factor de distorsión atañido es la ausencia de una codificación diagnóstica específica, en muchos de los casos, tras la primera entrevista de evaluación. Este aspecto afecta de manera importante a este estudio, pues los datos sobre el juicio diagnóstico de los pacientes nuevos se extraen del programa informático y no de la propia historia clínica. A pesar de estas limitaciones, consideramos que los resultados que se comentan a continuación pueden ser válidos para los objetivos de este trabajo.

A partir de los datos obtenidos podemos esbozar un perfil aproximado del paciente tipo que acudió a los Servicios de Salud Mental del distrito de Aranjuez durante el año 2004.

El paciente tipo procede de Aranjuez o Valdemoro $(68,9 \%)$, tiene menos de 45 años $(77,1 \%)$, es mujer $(57,6 \%)$, soltero/a (49\%) o casado/a (41,7\%), vive en pareja $(52,6 \%)$, con un nivel educativo inferior al nivel de E.G.B. $(59,2 \%)$, se encuentra en una situación laboral de retiro, jubilación y/o pensionista $(45,1 \%)$. En la población activa laboralmente, predominan las ocupaciones no bien especificadas $(42,5 \%)$ y el sector de la hostelería, servicios y seguridad $(36,8 \%)$. Algunas características de este paciente tipo (procedencia, edad, nivel educativo, ocupación) coinciden con las características sociodemográficas de la población del distrito (tabla 1). En este sentido, cabe destacar que el sexo femenino está presente en nuestra muestra en un porcentaje por encima del que le correspondería con arreglo a la población $(49,9 \%)$. Nos preguntamos si el sexo femenino es un factor de riesgo para el desarrollo de psicopatología o bien son posibles otras explicaciones que justifiquen esta predominancia, tan frecuente en la mayoría de estudios (7) sobre utilización de servicios de Salud Mental.

En el caso de los menores de 17 años un 77,4\% vive con ambos padres. En el caso de vivir con uno de los progenitores, la proporción es de $20,9 \%$ con la madre frente al $0,9 \%$ con el padre.

Algunos de los datos recogidos son muy concordantes con las características del distrito, esto es, con pequeños núcleos de población, algunos con menos de mil habitantes, lo que explicaría la masiva procedencia de los grupos de población más importantes, que tienen además una previsión de crecimiento mayor (Valdemoro y Aranjuez). Se caracteriza por ser un distrito de crecimiento poblacional receptor de población joven de otras procedencias, lo que se reflejaría en la baja edad de los pacientes que acudieron al Servicio. Las infraestructuras educativas, sociales y laborales son propias de la zona sur de la Comunidad de Madrid. Por ello, consideramos que el bajo nivel educativo, la situación laboral y el tipo de ocupación encontrados serían representativos del bajo nivel de desarrollo de la comarca (1). 
Tabla 7

Diagnósticos en función del sexo (\%)

\begin{tabular}{|c|c|c|c|c|}
\hline CÓDIGO & DIAGNÓSTICO & MUJERES & HOMBRES & TOTAL \\
\hline 290 & Psicosis orgánica senil y presenil & 1,1 & - & 0,6 \\
\hline 291 & Psicosis alcohólicas & 0,1 & - & 0,07 \\
\hline 292 & Psicosis debidas a drogas & 0,1 & 0,5 & 0,3 \\
\hline 293 & Psicosis orgánicas transitorias & - & - & - \\
\hline 294 & Otras psicosis orgánicas (crónicas) & 0,2 & 0,3 & 0,3 \\
\hline 295 & Psicosis esquizofrénica & 0,5 & 1,3 & 0,9 \\
\hline 296 & Psicosis afectivas & 3,9 & 3,6 & 3,8 \\
\hline 297 & Estados paranoides & 0,5 & 0,6 & 0,6 \\
\hline 298 & Otras psicosis no orgánicas & 0,6 & 0,6 & 0,6 \\
\hline 299 & Psicosis peculiares de la niñez & - & 0,3 & 0,1 \\
\hline 300 & Trastornos neuróticos & 30,9 & 17,1 & 25,0 \\
\hline 301 & Trastornos de la personalidad & 2,7 & 2,1 & 2,5 \\
\hline 302 & Desviaciones y trastornos sexuales & 0,3 & 1,6 & 0,9 \\
\hline 303 & Síndrome de dependencia del alcohol & 0,3 & 3,8 & 1,8 \\
\hline 304 & Dependencia de las drogas & 0,2 & 0,6 & 0,4 \\
\hline 305.0 & Alcohol (abuso) & 0,1 & 0,8 & 0,4 \\
\hline 305 & Abuso de drogas sin dependencia & 0,4 & 0,5 & 0,4 \\
\hline 306 & Alteraciones corp. orig. por factores mentales & 0,5 & 0,5 & 0,5 \\
\hline 307 & $\begin{array}{l}\text { Síntomas o síndromes especiales no clasificados en otra parte } \\
\text { Movimiento } \\
\text { Alimentación } \\
\text { Sueño } \\
\text { Esfínteres } \\
\text { Dolor }\end{array}$ & $\begin{array}{l}- \\
- \\
2,3 \\
0,8 \\
1,0 \\
0,1\end{array}$ & $\begin{array}{l} \\
1,0 \\
0,6 \\
0,8 \\
1,6 \\
0,3\end{array}$ & $\begin{array}{l}0,4 \\
1,6 \\
0,9 \\
1,3 \\
0,2\end{array}$ \\
\hline 308 & Reacción aguda ante gran tensión & 1,7 & 1,5 & 1,6 \\
\hline 309 & Reacción de adaptación & 23,3 & 21,4 & 22,5 \\
\hline 310 & Trast. Ment. no psicóticos por lesión orgánica encéfalo & - & - & - \\
\hline 311 & Trastornos depresivos no clasificados en otra parte & 2,1 & 2,5 & 2,2 \\
\hline 312 & Perturbación conducta no clasificada en otra parte & 0,6 & 1,9 & 1,0 \\
\hline 313 & Perturb. emociones propias de la niñez y adolescencia & 3,3 & 4,6 & 3,9 \\
\hline 314 & Síndrome hipercinético de la niñez & 0,5 & 2,1 & 1,9 \\
\hline 315 & Retardo selectivo del desarrollo & 0,5 & 0,3 & 0,5 \\
\hline 316 & $\begin{array}{l}\text { Factores psíquicos asociados a enf. clasf. en otra parte } \\
\text { Retraso mental } \\
\text { Sin especificar } \\
\text { No patología } \\
\text { Códigos V }\end{array}$ & $\begin{array}{r}0,3 \\
17,9 \\
1,9 \\
0,6\end{array}$ & $\begin{array}{r}- \\
0,6 \\
21,6 \\
1,6 \\
0,3\end{array}$ & $\begin{array}{r}- \\
0,6 \\
19,5 \\
1,8 \\
0,5\end{array}$ \\
\hline
\end{tabular}




\section{ORIGINALES Y REVISIONES}

Tabla 8

Diagnósticos en función de la edad (\%)

\begin{tabular}{|c|c|c|c|c|c|c|c|}
\hline CÓDIGO & DIAGNÓSTICO & $0-15$ & $16-30$ & $31-45$ & $46-60$ & $61-75$ & $76--$ \\
\hline 290 & Psicosis orgánica senil y presenil & - & - & - & - & 1,1 & 14,9 \\
\hline 291 & Psicosis alcohólicas & - & - & 0,2 & - & - & - \\
\hline 292 & Psicosis debidas a drogas & - & 0,7 & - & 0,5 & - & - \\
\hline 293 & Psicosis orgánicas transitorias & - & - & - & 0,5 & - & - \\
\hline 294 & Otras psicosis orgánicas (crónicas) & - & - & 0,2 & - & 1,1 & 2,1 \\
\hline 295 & Psicosis esquizofrénica & - & 0,7 & 1,4 & 1,5 & 1,1 & - \\
\hline 296 & Psicosis afectivas & 0,3 & 3,3 & 3,7 & 5,0 & 11,9 & 14,9 \\
\hline 297 & Estados paranoides & - & 0,3 & - & 1,0 & 2,4 & 6,4 \\
\hline 298 & Otras psicosis no orgánicas & - & 1,0 & 0,7 & 0,5 & 1,1 & 2,1 \\
\hline 299 & Psicosis peculiares de la niñez & 0,3 & - & - & - & - & - \\
\hline 300 & Trastornos neuróticos & 4,2 & 30,5 & 35,5 & 32,5 & 25,0 & 21,3 \\
\hline 301 & Trastornos de la personalidad & 0,3 & 5,4 & 2,8 & 3,5 & - & - \\
\hline 302 & Desviaciones y trastornos sexuales & - & 1,0 & 1,2 & 2,0 & 1,1 & - \\
\hline 303 & Síndrome de dependencia del alcohol & 0,3 & 1,0 & 2,6 & 6,0 & - & - \\
\hline 304 & Dependencia de las drogas & 0,3 & 0,3 & 0,7 & 0,5 & - & - \\
\hline 305.0 & Alcohol (abuso) & 0,3 & 0,3 & 0,4 & 0,5 & 2,4 & - \\
\hline 305 & Abuso de drogas sin dependencia & - & 1,0 & 0,2 & - & - & - \\
\hline 306 & $\begin{array}{l}\text { Alteraciones corporales orig. por } \\
\text { factores mentales }\end{array}$ & 0,7 & - & 1,4 & 0,5 & - & - \\
\hline 307 & $\begin{array}{l}\text { Síntomas o síndromes especiales no } \\
\text { clasificados en otra parte } \\
\text { Movimiento } \\
\text { Alimentación } \\
\text { Sueño } \\
\text { Esfínteres } \\
\text { Dolor } \\
\end{array}$ & $\begin{array}{l}- \\
1,3 \\
3,9 \\
3,3 \\
6,2 \\
0,3\end{array}$ & $\begin{array}{l}- \\
0,3 \\
3,7 \\
0,3 \\
0,3 \\
0,6\end{array}$ & $\begin{array}{l}- \\
- \\
0,7 \\
- \\
- \\
-\end{array}$ & $\begin{array}{l}- \\
- \\
- \\
- \\
- \\
-\end{array}$ & $\begin{array}{l}- \\
- \\
- \\
3,6 \\
- \\
-\end{array}$ & $\begin{array}{l}- \\
- \\
- \\
- \\
- \\
-\end{array}$ \\
\hline 308 & Reacción aguda ante gran tensión & 1,3 & 3,7 & 1,2 & 1,0 & 1,1 & - \\
\hline 309 & Reacción de adaptación & 23,2 & 24,2 & 25,2 & 24,5 & 21,4 & 6,4 \\
\hline 310 & $\begin{array}{l}\text { Trast. ment. no psicóticos por lesión } \\
\text { orgánica de encéfalo }\end{array}$ & - & - & - & - & - & - \\
\hline 311 & $\begin{array}{l}\text { Trastornos depresivos no clasificados } \\
\text { en otra parte }\end{array}$ & 0,3 & 1,3 & 2,6 & 4,5 & 2,4 & 8,5 \\
\hline 312 & $\begin{array}{l}\text { Perturbación conducta no clasificada } \\
\text { en otra parte }\end{array}$ & 3,6 & 0,6 & - & 0,5 & - & - \\
\hline 313 & $\begin{array}{l}\text { Perturb. emociones propias niñez y } \\
\text { adolescencia }\end{array}$ & 18,0 & 1,3 & - & - & - & - \\
\hline 314 & Síndrome hipercinético de la niñez & 10,5 & - & - & - & - & - \\
\hline 315 & Retardo selectivo del desarrollo & 1,3 & 0,3 & - & - & - & - \\
\hline 316 & $\begin{array}{l}\text { Factores psiq. asociados a enf. clasificadas } \\
\text { en otra parte } \\
\text { Retraso mental } \\
\text { Sin especificar } \\
\text { No patología } \\
\text { Códigos V }\end{array}$ & $\begin{array}{r}- \\
0,7 \\
14,1 \\
5,2 \\
-\end{array}$ & $\begin{array}{r}- \\
0,3 \\
12,8 \\
3,3 \\
1,0\end{array}$ & $\begin{array}{r}- \\
0,4 \\
16,1 \\
2,6 \\
-\end{array}$ & $\begin{array}{r}- \\
- \\
12,7 \\
2,0 \\
0,5\end{array}$ & $\begin{array}{r}- \\
1,1 \\
21,4 \\
1,1 \\
-\end{array}$ & $\begin{array}{c}- \\
- \\
23,4 \\
- \\
-\end{array}$ \\
\hline
\end{tabular}


A los autores de este estudio nos llama la atención el elevado porcentaje de pacientes incluidos en la categoría de retirado, jubilado y pensionista $(45,1 \%)$ en clara discrepancia con las otras variables y el contacto diario con los pacientes, así como con las características de la población. Pensamos que tras estos datos puede haber un error de codificación que habría que investigar.

Si tenemos en cuenta la variable sexo como moduladora de los datos sociodemográficos, obtenemos dos perfiles diferenciados en algunas variables. En el caso del hombre, procede de Valdemoro o Aranjuez (66\%), es menor de 45 años $(78,3 \%)$, predominantemente soltero $(58,2 \%)$, que, en el caso de los adultos, convive con su cónyuge $(50,2 \%)$ y, en cuanto al nivel educativo, no alcanza el nivel de E.G.B. en un $81,8 \%$. En el caso de la mujer, hay también una masiva procedencia de Valdemoro o Aranjuez (71\%), es menor de 45 años (75,1\%), y desde el punto de vista de su estado civil no hay una única categoría que predomine, siendo las casadas un $46,1 \%$ frente al $42,2 \%$ de solteras. En la convivencia, adultos, hay un predominio de la modalidad con cónyuge (54\%). Respecto al nivel educativo no se alcanza el nivel de E.G.B. en un 79\%.

Respecto a las variables sexo, estado civil, tipo de convivencia, nivel educativo y situación laboral, hemos encontrado resultados similares en estudios de muy diversa procedencia $(5 ; 6 ; 8 ; 9 ; 10 ; 11 ; 12)$. Sin embargo, otros trabajos contradicen algunos de estos resultados, por ejemplo en el estudio realizado en la población atendida por el CSM de Hortaleza (13) los sexos están equiparados en cuanto a los pacientes nuevos. En el estudio del área rural de Xátiva (Valencia) (14), así como en el de Encartaciones (Vizcaya) (15) y el efectuado sobre toda la provincia de Segovia (7) la edad de los pacientes nuevos es mayor. Una explicación de esta disparidad de resultados es que las variables sociodemográficas influyen de forma diferente en función de las características de cada comunidad. Es posible imaginar que algunas de estas diferencias tengan que ver con las características de las rutas asistenciales y la organización de los servicios (accesibilidad, disponibilidad, ofertas diferenciadas en cuanto a tratamientos, etc.).

En cualquier caso conviene recordar que este estudio, como cualquier otro, no puede ser un reflejo directo del estado de salud mental de la zona atendida. Existen estudios $(6 ; 16)$ que señalan que los patrones de utilización de los Servicios de Salud Mental están mediados por procesos intrapsíquicos, así como sociales y culturales.

La variable sexo no arroja diferencias llamativas en cuanto a las variables procedencia, convivencia y nivel educativo. En el resto de las variables sí cabe mencionar algunas diferencias, esto es, en la edad la mayor diferencia se produce en el grupo de 0-15 años (3\% hombres frente 19\% mujeres). Este dato es congruente con otros trabajos $(12)$, y con la literatura científica $(17 ; 18)$ que 
expone la predominancia de varones en la demanda psiquiátrica en los primeros años de la vida. Esta situación se invierte a partir de la pubertad y adolescencia (en la franja de 16-30 se produce un aumento de la demanda: $24,3 \%$ de mujeres frente al $17,1 \%$ de los varones), llegando a igualarse prácticamente en la franja de 31-45.

Respecto al estado civil los datos hallados son congruentes con algunos de los estudios consultados $(6 ; 8 ; 12)$, pues predomina la soltería en los varones.

$\mathrm{Si}$ atendemos en primer lugar al total de los pacientes nuevos (tabla 7) sin distinción de sexo o edad, encontramos que las categorías diagnósticas más frecuentes son «Trastornos neuróticos» (25\%) y «Reacciones de adaptación» $(22,5 \%)$. En un $19,5 \%$ de casos no se produce una asignación de diagnóstico tras la primera entrevista de evaluación. Estos datos coinciden con la mayoría de los estudios revisados por los autores de este trabajo.

Otros diagnósticos frecuentes son: «Perturbaciones de las emociones peculiares de la niñez y adolescencia» (3,9\%), «Psicosis afectivas» $(3,8 \%)$, «Trastornos de la personalidad» $(2,5 \%)$, «Trastornos depresivos no clasificados en otra parte» $(2,2 \%)$, «Síndrome hipercinético» $(1,9 \%)$, «Síndrome de dependencia del alcohol» $(1,8 \%)$, «Trastornos de la alimentación» $(1,6 \%)$ y «Reacción aguda ante gran tensión» $(1,6 \%)$. En un $1,8 \%$ del total de casos no se apreció patología psiquiátrica alguna. En el 0,9\% de casos se diagnosticó en primer lugar «Psicosis esquizofrénica».

Si tenemos en cuenta la variable sexo (tabla 7) encontramos que en las mujeres predominan las siguientes categorías: «Trastornos neuróticos» $(30,9 \%)$ y «Reacciones de adaptación» (23,3\%). En un 17,9\% no se especifica ningún diagnóstico.

En el caso de los hombres, alcanzan cifras semejantes el grupo de los que no tienen un diagnóstico específico (21,6\%), y los diagnosticados de «Reacciones de adaptación» (21, 4\%). En tercer lugar habría que situar los «trastornos neuróticos» $(17,1 \%)$.

Existen algunas categorías diagnósticas en las que hemos podido observar marcadas y llamativas diferencias en función del sexo. En «Trastornos de la alimentación» el porcentaje entre las mujeres es cuatro veces superior al que existe entre los hombres. En «Trastornos neuróticos» el porcentaje de mujeres es el doble que en los hombres. En «Dependencia del alcohol» casi no hay casos de mujeres, frente a un $3,8 \%$ de hombres. En «Psicosis esquizofrénica», en cambio, el porcentaje de hombres casi triplica al de mujeres. En «Síndrome hipercinético» lo cuadruplica. En «Psicosis orgánicas» la categoría diagnóstica no aparece en varones. En «Desviaciones y trastornos sexuales», prácticamente no se recibe demanda entre las mujeres. 
En el resto de categorías diagnósticas las diferencias, cuando existen, no son tan marcadas.

La edad es otra de las variables que el R.A.C. permite estudiar en cuanto a la distribución de los diagnósticos. Son seis los grupos etarios que el programa informático permite (tabla 8).

En el grupo de cero a quince años, prácticamente la cuarta parte son «Reacciones de adaptación». La categoría «Perturbación de las emociones propias de la niñez y adolescencia» origina un $18 \%$ de casos. Existe un $14,1 \%$ de casos $\sin$ especificar. El «Síndrome hipercinético» aporta el 10\% de este grupo etario.

En el grupo de dieciséis a treinta años, el grupo de «trastornos neuróticos» es la categoría diagnóstica dominante (30\%), seguida de las «Reacciones de adaptación» $(24,2 \%)$. Un $12,8 \%$ de casos no resultan diagnosticados en la primera consulta en esta franja de edad.

En las tres franjas de edad siguientes se repite esta misma distribución en cuanto a las categorías diagnósticas de «Trastornos neuróticos», «Reacciones de adaptación» y «Sin especificar».

A partir de los setenta y seis años la categoría más frecuente es «Sin especificar», seguido de cerca por los «Trastornos neuróticos» (21,3\%). Destacan dos diagnósticos que rozan el 15\%: «Psicosis afectivas» $\mathrm{y}$ «Psicosis orgánicas».

Si analizamos las categorías diagnósticas a lo largo de los grupos etarios, observamos que los casos de «Psicosis esquizofrénicas» se concentran en las edades intermedias, mientras que en las «Psicosis afectivas» tenemos un incremento progresivo desde la infancia. En los «Estados paranoides» pensamos que la concentración en las dos últimas etapas vitales pudieran denotar problemas de diagnóstico diferencial con las psicosis orgánicas. En los «Trastornos neuróticos» se produce un descenso en el porcentaje a partir de los sesenta años. A partir de esta edad también desaparecen los siguientes diagnósticos: Trastornos de la personalidad, Dependencia de alcohol y drogas, Abuso de drogas, Problemas psicosomáticos, Trastornos de la alimentación, del Movimiento y Dolor.

El diagnóstico «Abuso de alcohol» se mantiene estable en las diferentes franjas, hasta los sesenta y un años, en que experimenta un importante aumento $(2,4 \%)$.

Los «Trastornos de Alimentación» aparecen como propios de los menores de treinta años, pues la cifra es muy baja en el grupo de treinta y uno a cuarenta y cinco y desaparece en las siguientes franjas.

Las «Reacciones de adaptación» ofrecen cifras similares en todas las franjas, excepto en mayores de setenta y seis años, decreciendo notablemente. Creemos que este descenso es compensado por el incremento en «Psicosis afectivas».

Es significativo que la categoría «Síndrome hipercinético de la niñez» desaparece por encima de los quince años. La categoría «Sin especificar», al contra- 
rio, aumenta con la edad, de modo que en los dos últimos grupos supera el $20 \%$. Por otro lado, los resultados señalan que se tiende a diagnosticar menos patología en las franjas de menor edad.

Los resultados obtenidos, al analizar los diagnósticos en función del sexo y la edad, son congruentes básicamente con la literatura epidemiológica general (19; $20 ; 21)$. En relación a la población española, los trabajos que hemos hallado, indagan con profundidad en las características sociodemográficas de la muestra, pero hemos echado en falta un análisis en detalle de las diferentes categorías diagnósticas y su variación en función de diferentes variables (sexo, edad, etc.).

\section{BIBLIOGRAFÍA}

(1) Instituto de Estadística de la Comunidad de Madrid, Reseña estadística zonal 2005, Madrid, Comunidad de Madrid, 2005.

(2) ElPers, J. R., «Community Psychiatry», en KAPlan y SADOcK (ed.), Comprehensive Textbook of Psychiatry, Baltimore, Williams and Wilkins, 1995.

(3) Comunidad de Madrid. Consejería de Sanidad y Servicios Sociales, Programas de los Servicios de Salud Mental, Madrid, Publicaciones del Servicio Regional de Salud, 1989.

(4) Organización Mundial de la Salud, Clasificación Estadística Internacional de las Enfermedades, Lesiones y Causas de Muerte, Ginebra, O.M.S., 1977, novena revisión.

(5) Martínez, O., y otros, «Estudio de las primeras consultas de un centro de Salud Mental (I). Características sociodemográficas, vías de derivación y tratamiento anterior», Revista de la AEN, 1997, XVII, 61, pp. 37-60.

(6) Montilla, J. F., y otros, «Uso de servicios ambulatorios de Salud Mental en España. Consumo de recursos en el primer año de asistencia a pacientes nuevos», Revista de la AEN, 2002, XXI, 84, pp. 25-47.

(7) De Francisco Beltrán, P.; Goenaga Palancares, E., «Diferencias de la demanda en Salud Mental según hábitat», Revista de la AEN, 2002, XXII, 82, pp. 105-113.

(8) Dorado Primo, J. A., y otros, «Análisis de la demanda en el equipo de salud mental de Andújar. Evolución y estudio comparativo a los 20 años de apertura», Revista de la AEN, 2002, XXVII, 84, pp. 141-154.

(9) Balmón Cruz, C.; Dorado Primo, J. A., «Primeros ingresos en el hospital psiquiátrico y unidades de hospitales generales», Psiquis, 1995, 16, 7, pp. 41-53.

(10) RodríGuez, M.; Almeida, A.; VAle, M., «Indicadores de morbilidad asistida en la Unidad de Salud Mental de Santa Cruz de la Palma en 1988-89», Rev. A. Psiquiatría, 1992, 8, 1, pp. $15-20$.

(11) Castaño, C., y otros, «Utilización de los servicios de salud mental de Alcalá de Henares», Revista de la AEN, 1991, 39, pp. 289-97.

(12) Ruiz Doblado, S.; Pérez Cano, A.; Vicente Ortiz, A., «Trastornos psiquiátricos en un área rural: estudio descriptivo de 2.016 casos», Actas Luso-Esp. Neurol. Psiquiatr., 1997, 25, 1, pp. 23-33.

(13) Vicente, N.; EstéveZ, L.; JUÁREZ, E., «Análisis de la demanda asistencial en un Centro de Salud Mental», Arch. Neurobiol., 1990, 53, pp. 13-7.

(14) Arribas, P., y otros, «Análisis de la evolución de la demanda asistencial en un Centro de Salud Mental», Actas Luso-Esp. Neurol. Psiquiatr., 1994, 22, pp. 1-5.

(15) Peralta, C.; Goiriena, D.; Salcedo, B., «Estudio descriptivo de la actividad asistencial anual de un consultorio de salud mental de nueva creación», An. Psiquiatr., 1994, 10, pp. 314-9.

(16) Howard, K. I., y otros, «Patterns of Mental Health Service Utilization», Arch. Gen. Psychiatry, 1956, 53, pp. 696-703. 
(17) OFFORD, D. R., «Epidemiología de los trastornos psiquiátricos en la infancia y la adolescencia», en GELDER, M., y otros (eds.), Tratado de psiquiatría, Barcelona, Ars Medica, 2003.

(18) RutTer, M., «Development and Psychopathology», en RutTer, M.; TAYlor, E. (eds.), Child and Adolescent Psychiatry, Oxford, Blackwell, 2002.

(19) American Psychiatric Association, DSM-IV-TR. Manual diagnóstico y estadístico de los trastornos mentales, texto revisado, Barcelona, Masson, 2002.

(20) REgIER, D. A.; BURKE, J. D., «Métodos cuantitativos y experimentales en Psiquiatría», en KaPlan y SADOcK (eds.), Tratado de psiquiatría, Buenos Aires, Intermédica, 1997.

(21) Henderson, S., «Contribución de la Epidemiología a la Etiología Psiquiátrica», en Gelder, M., y otros (eds.), Tratado de psiquiatría, Barcelona, Ars Medica, 2003.

* Juan José Belloso Ropero, psicólogo clínico, Centro de Salud Mental de Ciempozuelos, Madrid; José Carlos Espín Jaime, psiquiatra, Centro de Salud Mental de Valdemoro, Madrid. Correspondencia: Juan José Belloso Ropero. Complejo Asistencial Benito Menni, C/ Jardines, 128350, Ciempozuelos (Madrid); jjbelloso@ hotmail.com

** Fecha de recepción: 11-IX-2006. 